\title{
Polymerbasierte Kompositmaterialien für Festelektrolytsensoren
}

\author{
Corinna Vonau $^{1)}$, Jens Zosel ${ }^{2)}$, Muthusamy Paramasivam ${ }^{3)}$, Kristina Ahlborn ${ }^{2)}$, Frank Gerlach ${ }^{2)}$, Jan
} Poppe $^{4)}$, Ulrich Guth ${ }^{2,4)}$

1) Universität Leipzig, Institut für Analytische Chemie, Johannisallee 29, 04103 Leipzig

${ }^{2)}$ Kurt-Schwabe-Institut für Mess- und Sensortechnik e.V. Meinsberg, Kurt-Schwabe-Straße 4, 04720 Ziegra-Knobelsdorf

3) Central Electrochemical Research Institute, Karaikudi 630 006, Tamil Nadu, India

4) Technische Universität Dresden, Institut für Physikalische Chemie und Elektrochemie, Bergstraße 66b, 01062 Dresden

\section{Zusammenfassung}

Festelektrolyt-Gassensoren auf der Basis von Yttriumoxid-stabilisiertem Zircondioxid (YSZ) und ausgestattet mit Mischpotentialelektroden kommen zur potentiometrischen Bestimmung geringer Konzentrationen verschiedener brennbarer Gase wie Wasserstoff, Kohlenmonoxid und Kohlenwasserstoffen in sauerstoffhaltigen Gasphasen zum Einsatz [1]. Sensitivität, Selektivität und Langzeitstabilität dieser Sensoren werden entscheidend durch das Elektrodenmaterial bestimmt [2], wobei diese Parameter für eine Vielzahl potentieller Applikationen bislang noch nicht die notwendigen Anforderungen erfüllen. Weltweit wird deshalb intensiv an der Entwicklung neuer Elektrodenmaterialien gearbeitet, die möglichst leicht zugänglich, umweltfreundlich und kostengünstig sein sollen. Daher wurde eine Gruppe neuer Materialien auf der Basis leitfähiger Polymerkomposite hergestellt, die erstmals im Hinblick auf ihren Einsatz als Mischpotentialelektroden in Festelektrolyt-Gassensoren untersucht werden konnte.

\section{Einleitung}

Elektrochemische Festelektrolyt-Gassensoren dienen der zuverlässigen, sensitiven und schnellen Messung kleinster Konzentrationsbereiche verschiedener brennbarer Gase im Hochtemperaturbereich [3]. Dabei sind die Sensorparameter Sensitivität, Selektivität und Langzeitstabilität entscheidend von dem Elektrodenmaterial einer Mischpotentialelektrode abhängig [2]. Trotz zahlreicher Bemühungen in den letzten zwei Jahrzehnten [4-6] gibt es für diese Sensorparameter noch erheblichen Verbesserungsbedarf, da die Anforderungen für eine Vielzahl potentieller Applikationen noch nicht erfüllt werden. Größere Defizite bestehen oftmals bei der Langzeitstabilität [7, 8], die für Messelektroden aus Gold oder anderen Edelmetallen bei Messungen über mehrere Monate ohne zwischenzeitliche Kalibrierung selten gegeben ist. Daher ist die Suche nach neuen leitfähigen Materialien für Komposite zur Herstellung von Mischpotentialelektroden sinnvoll, wobei diese die notwendig hohe elektrochemische Aktivität, thermische Stabilität und geringe katalytische Aktivität gewährleisten müssen. Von Vorteil bei Synthese und Untersuchung neuer Materialien sind eine leichte und kostengünstige Zugänglichkeit der Materialien, sowie deren Stabilität und Umweltfreundlichkeit. Polyanilin (PANI) als eines der stabilsten der intrinsisch leitfähigen Polymere erfüllt diese Anforderungen in hohem Maß [9] und wurde deshalb für die Synthese einer neuen Gruppe von Kompositen unter Beimengung verschiedener Metalloxide und Metallsalze wie $\mathrm{Nb}_{2} \mathrm{O}_{5}$ bzw. $\mathrm{FeCl}_{3}$ und $\mathrm{Co}\left(\mathrm{NO}_{3}\right)_{2}$ ausgewählt. Diese neu entwickelten Komposite wurden mittels Transmissionselektronenmikroskop (TEM), Rasterelektronenmikroskop (REM), thermischer Analyse (TG/DTA), Leitfähigkeitsmessungen und katalytischen Untersuchungen charakterisiert und im Hinblick auf ihren Einsatz als Mischpotentialelektroden in Festelektrolyt-Gassensoren bei $450{ }^{\circ} \mathrm{C}$ für Wasserstoff und die Kohlenwasserstoffe Propen und Ethen untersucht. 


\section{Experimentelles}

Wie in Tafel 1 beschrieben wurden sechs Proben mittels oxidativer Synthese hergestellt, wobei die in reinem PANI resultierenden Proben 1 und 2 als Referenz dienen und sich durch die eingesetzten Mengen der Ausgangsstoffe unterscheiden. Die Proben 3 und 4 enthalten zusätzlich das Metalloxid $\mathrm{Nb}_{2} \mathrm{O}_{5}$, wobei das $\mathrm{Nb}_{2} \mathrm{O}_{5}$ bei Probe 3 vor Beginn der Polymerisation, bei Probe 4 nach Beginn der Polymerisation zugegeben wurde. Die Proben 1 bis 4 wurden nach ihrer Synthese intensiv über mehrere Tage gewaschen, um die Salze des Oxidationsmittels und der Salzsäure nahezu vollständig zu entfernen. Als Endpunktanzeige für diese Prozedur wurde der in Tafel 1 angegebene pH-Wert des Waschwassers herangezogen. Probe 5 wurde auf gleiche Weise wie Probe 4 hergestellt, jedoch nach der Synthese nur bis zu einer Farbänderung gewaschen. Auch bei Probe 6 wurde der Waschvorgang bei erheblich kleineren pH-Werten abgebrochen, diese enthält im Gegensatz zu Probe 5 kein Metalloxid, sondern die Salze $\mathrm{FeCl}_{3}$ und $\mathrm{Co}\left(\mathrm{NO}_{3}\right)_{2}$.

Tafel 1 Parameter der verschiedenen Proben für die Synthese.

\begin{tabular}{c|c|c|c|c|c}
\hline Probennummer & $\begin{array}{c}\text { Anilin } \\
\text { [mol] }\end{array}$ & $\begin{array}{c}\text { Ammoniumper- } \\
\text { oxodisulfat [mol] }\end{array}$ & $\begin{array}{c}\mathrm{Nb}_{2} \mathrm{O}_{5} \\
{[\mathrm{~mol}]}\end{array}$ & $\begin{array}{c}\mathrm{FeCl}_{3} / \mathrm{Co}\left(\mathrm{NO}_{3}\right)_{2} \\
{[\mathrm{~mol}]}\end{array}$ & Waschgang \\
\hline 1 & 0,1 & 0,05 & 0 & 0 & \multirow{2}{*}{ Waschwasser $\approx$} \\
$\mathrm{pH} 4$ \\
\hline 2 & 0,4 & 0,1 & 0 & 0 & 0 \\
\hline 4 & 0,1 & 0,05 & 0,05 & 0 & Produkt blau \\
\hline 5 & 0,1 & 0,05 & 0,05 & 0 & Waschwasser \\
farblos
\end{tabular}

In Abhängigkeit von der Aufarbeitung lassen sich verschiedene Formen von PANI darstellen, die sich in ihren Oxidationsformen unterscheiden (Bild 1). Eine intrinsische Leitfähigkeit erreicht PANI nur in der Form des protonierten Emeraldinsalzes 4. Die Proben 1 bis 4 bestehen daher aus einer Mischung aus neutraler Emeraldin-Base 5 und leitfähigem Emeraldinsalz 4 und die Proben 5 und 6 ausschließlich aus dem leitfähigen Salz 4.

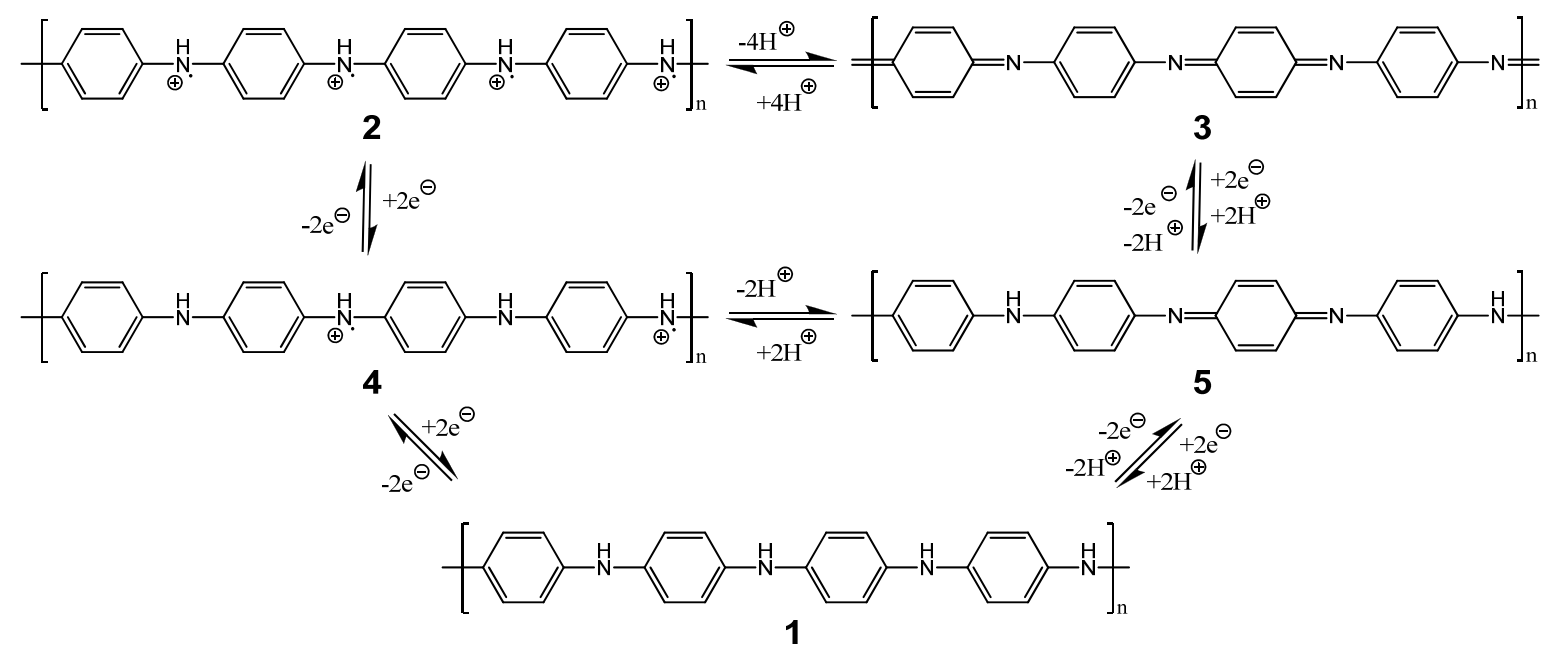

Bild 1 Oxidationsformen von Polyanilin (PANI): 1 Leucomeraldin, 2 protoniertes Pernigranilin(-salz), 3 PernigranilinBase, 4 protoniertes Emeraldin(-salz), 5 Emeraldin-Base.

Nach der Synthese wurden die Proben hinsichtlich ihrer Struktur und Morphologie, thermischen Stabilität, Leitfähigkeit, katalytischen Aktivität sowie ihrer Sensitivität mittels potentiometrischer Messungen in Gasgemischen mit Wasserstoff, Propen und Ethen untersucht. Für die potentiometrischen Untersuchungen wurden aus den hergestellten Proben Pasten mit Hilfe eines kommerziellen Binders auf Epoxidharzbasis (Binder A) und alternativ mit einem Binder aus acetonhaltiger Acetylcellulose (Binder B) hergestellt, mittels Dickschichttechnik auf YSZ-Scheiben aufgedruckt und bei $150{ }^{\circ} \mathrm{C}$ für $1 \mathrm{~h}$ getrocknet. 


\section{Ergebnisse}

\subsection{Struktur und Morphologie}

Die TEM-Aufnahme in Bild 2a belegt den kristallinen Charakter des reinen PANI anhand von parallelen Linien in den Makromolekülen. Dieser ist auch bei den Kompositproben in Bild 2b und c sichtbar, wobei die unterschiedlichen Zeitpunkte der Zugabe von $\mathrm{Nb}_{2} \mathrm{O}_{5}$ vor bzw. nach Beginn der Polymerisation in einer relativ gleichmäßigen kristallinen Hülle aus PANI um den Metalloxidpartikel bei Probe 3 und der unregelmäßigen Anlagerung von Makromolekülen ohne Hüllenbildung bei Probe 4 resultieren. Es ist demnach davon auszugehen, dass bei Probe 3 die einzelnen Anilinmonomere schrittweise an das Metalloxid adsorbieren und dort polymerisieren, während bei Probe 4 auspolymerisierte Partikel sich an die Metalloxidpartikel anlagern.

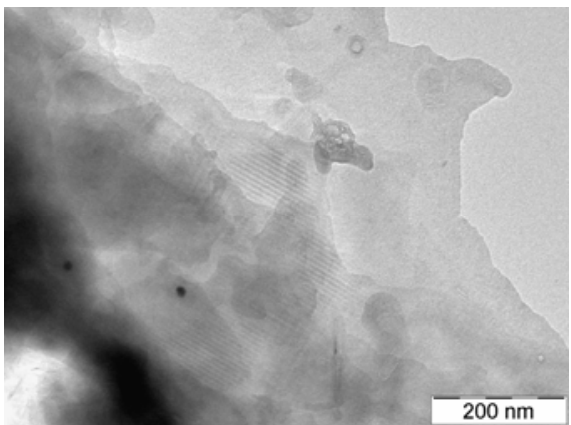

a)

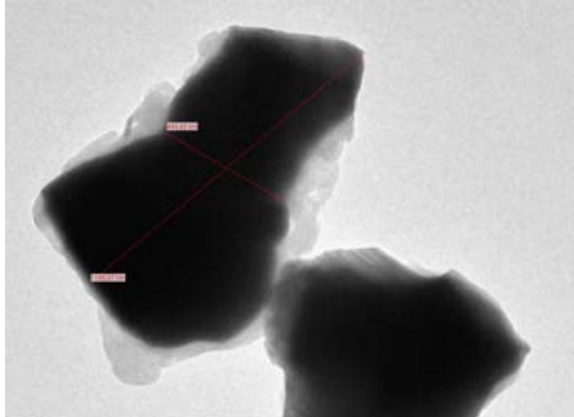

b)

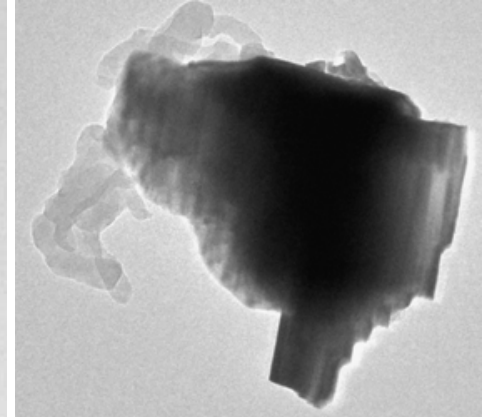

c)

Bild 2 TEM-Aufnahmen. a) Reines PANI. b) Probe $3\left(\mathrm{Nb}_{2} \mathrm{O}_{5}\right.$ wurde vor Polymerisationsbeginn zugegeben). c) Probe 4 $\left(\mathrm{Nb}_{2} \mathrm{O}_{5}\right.$ wurde nach Polymerisationsbeginn zugegeben).

Die reinen PANI-Proben wirken in den REM-Aufnahmen gröber und agglomerierter als die metalloxidhaltigen Kompositproben. Bei dem metallsalzhaltigen Komposit ist visuell kein Unterschied zum reinen PANI zu erkennen. Nach der thermischen Analyse bis $500{ }^{\circ} \mathrm{C}$ wirken die Partikel aller Proben kleiner, wobei deren Farbe belegt, dass noch intaktes PANI darin enthalten ist.

\subsection{Thermische Analyse}

Die thermische Analyse wurde bis zu einer Temperatur von $500{ }^{\circ} \mathrm{C}$ durchgeführt. Bereits bei $400{ }^{\circ} \mathrm{C}$ tritt bei dem reinen Polymer ein großer Masseverlust auf, so dass letztlich mehr als $90 \%$ der reinen PANI-Proben 1 und 2 zersetzt wurden (Bild 3a). Die metalloxidhaltigen Proben zeigen demgegenüber eine deutliche thermische Stabilisierung, so dass ihr Masseverlust bei $500{ }^{\circ} \mathrm{C}$ auf $12 \%$ begrenzt wird (Bild $3 \mathbf{b}$ ). Da dieser Masseverlust ausschließlich den Anteil an PANI im Komposit betrifft, ergibt sich aus dem Mischungsverhältnis, dass nach der thermischen Behandlung noch etwa $71 \%$ PANI im Komposit vorhanden sind.
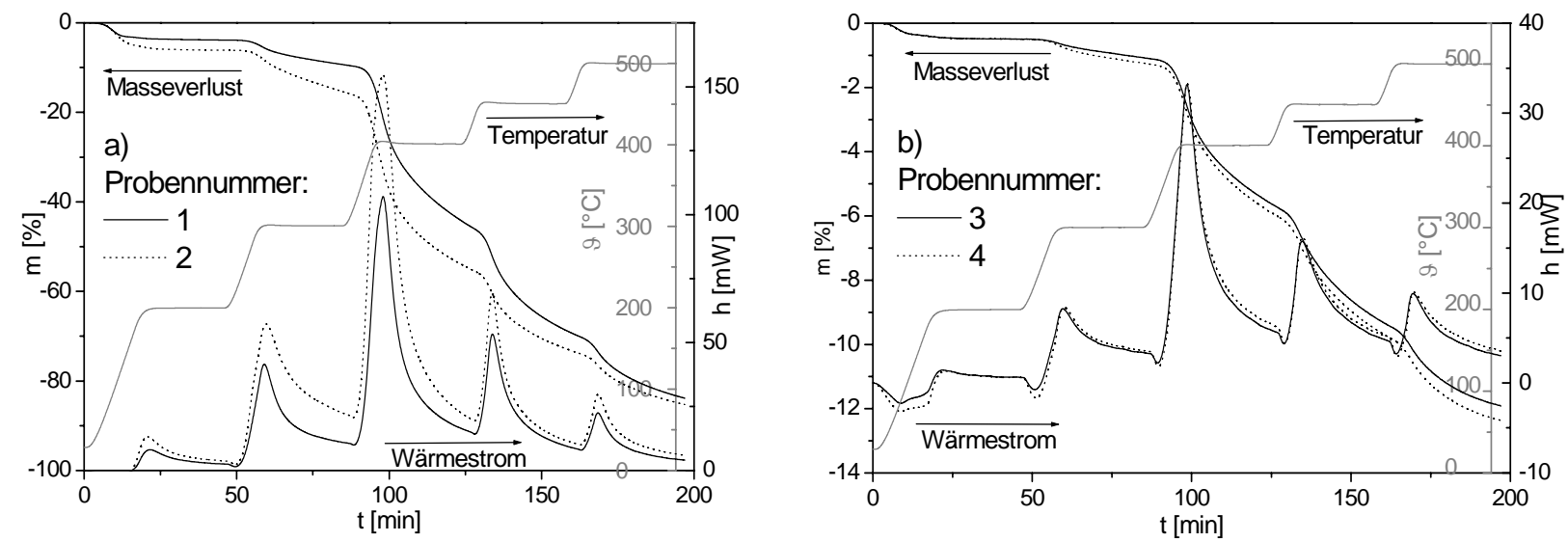

Bild 3 Thermische Analyse, a) Probe 1 und 2, b) Probe 3 und 4. 
Die Ursachen für diese thermische Stabilisierung konnten noch nicht aufgeklärt werden. Es ist jedoch zu vermuten, dass die kristalline Polymerstruktur aufbricht und sich die reaktiven Kettenenden stabilisierend an das Metalloxid binden. Die metallsalzhaltige Kompositprobe 6 zeigt dementsprechend auch einen ähnlich hohen Masseverlust wie die Proben 1 und 2.

\subsection{Potentiometrische Untersuchungen}

Die potentiometrischen Untersuchungen erbrachten im Rahmen auswertbarer Signal-Rausch-Verhältnisse nur für die sauer aufgearbeiteten Proben 5 und 6 deutliche Sensitivitäten wobei ein Anstieg dieses Parameters nicht wie bei anderen Elektrodenmaterialien mit sinkenden katalytischen Aktivitäten korreliert ist. Dies ist auf große Unterschiede der Leitfähigkeit zurückzuführen, die bei den Proben 5 und 6 etwa zwei Zehnerpotenzen höher ist als bei den Proben 3 und 4. Die jeweiligen Sensitivitäten der Proben bei einer Prüfgaskonzentration von 20 ppm sind in Bild 4 dargestellt. Appliziert mit dem Binder auf Epoxidharzbasis, erzielte Probe 6 größere Sensitivitäten als Probe 5. Da die mechanische Stabilität der mit diesem Binder hergestellten Elektroden für einen längerfristigen Einsatz noch nicht ausreicht, wurde Probe 5 mit einem Binder basierend auf acetonhaltiger Acetylcellulose (Binder B) getestet. Dieser führte zwar zu einer erhöhten Sensitivität, liefert jedoch ebenfalls keine höhere mechanische Stabilität des Elektrodenmaterials auf YSZ.
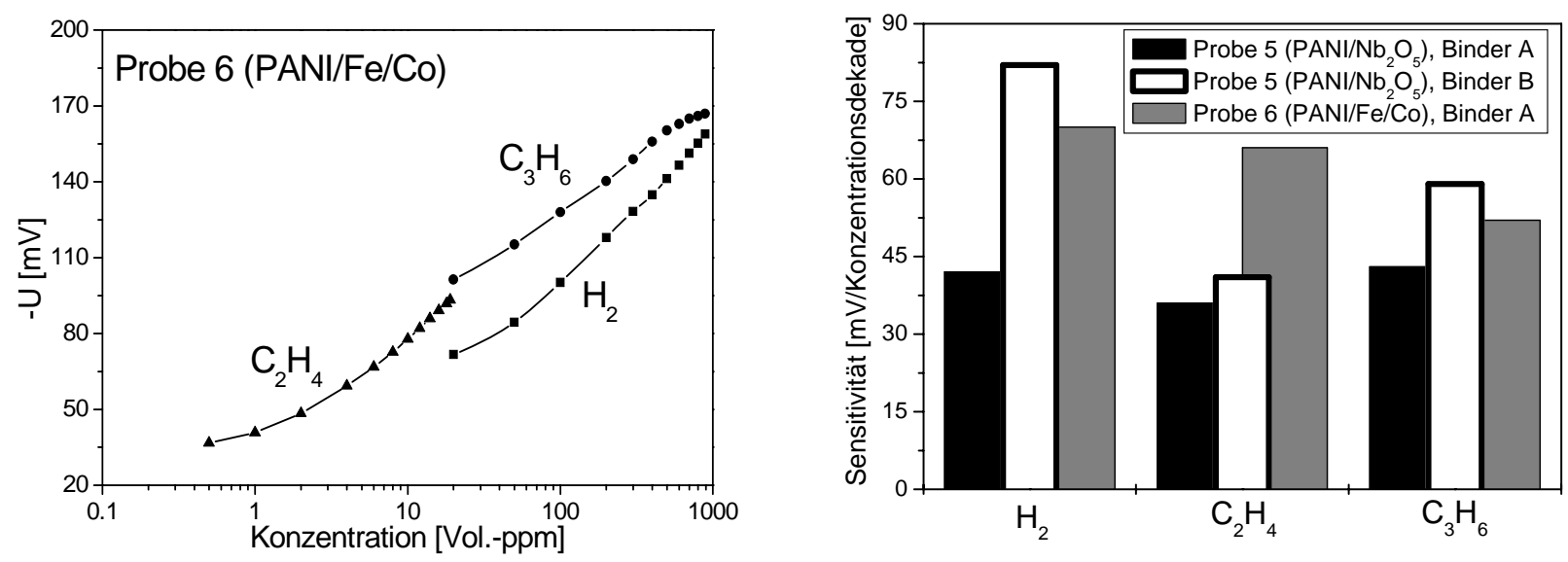

Bild 4 a) Potentialverlauf an einer Elektrode aus Probe 6 gegen eine Pt-Luft-Referenzelektrode bei $450{ }^{\circ} \mathrm{C}$ in Gemischen verschiedener oxidierbarer Gase, b) Sensitivitäten der Proben 5 und 6 im Vergleich

\section{Schlussfolgerung}

Erstmalig konnten Elektrodenmaterialien auf der Basis leitfähiger Polymere im Komposit mit $\mathrm{Nb}_{2} \mathrm{O}_{5}$ bzw. $\mathrm{FeCl}_{3} / \mathrm{Co}\left(\mathrm{NO}_{3}\right)_{2}$ für Festelektrolyt-Gassensoren geschaffen und bei Temperaturen von $450{ }^{\circ} \mathrm{C}$ über einen längeren Zeitraum untersucht werden. Diese Komposite ermöglichten die Bestimmung der Gase Wasserstoff, Propen und Ethen mit überraschend hohen Sensitivitäten von bis zu 80 mV/Konzentrationsdekade.

\section{Literatur}

[1] J. Zosel, D. Westphal, S. Jakobs, R. Müller, U. Guth, Solid State Ionics 152-153 (2002), 525-529.

[2] J. Zosel, D. Franke, K. Ahlborn, F. Gerlach, V. Vashook, U. Guth., Sens. Actuators 179 (2008) 16281631.

[3] V. Plashnitsa, T. Ueda, P. Elumalai, N. Miura Sens. Actuators B 130 (2008) 231-239.

[4] N. Miura, T. Raisen, G. Lu, N. Yamazoe, Sens. Actuators B 47, (1998) 84-91.

[5] F. H. Garzon, R. Mukundan, E. L. Brosha, Solid State Ionics 136-137, (2000) 633-638.

[6] S. Käding, S. Jakobs, U. Guth, Ionics 9 (2003) 151-154.

[7] E. L. Brosha, R. Mukundan, R. D. Brown, F. H. Garzon, Sens. Actuators B87 (2002) 47-57.

[8] D. Schönauer, K. Wiesner, M. Fleischer, R. Moos, Sens. Actuators B140 (2009) 585-590.

[9] A. Pud, N. Ogurtsov, A. Korzhenko, G. Shapoval, Prog. Polym. Sci. 28 (2003) 1701-1753. 